\title{
Metabolism of Brain Glycolipid Fatty Acids ${ }^{1,2}$
}

\author{
Yasuo Kishimoto and Norman S. Radin, Mental Health Research Institute,
} University of Michigan, Ann Arbor, Michigan

\begin{abstract}
The metabolism of the fatty acid moieties of brain cerebrosides, sulfatides, and gangliosides is reviewed and diseussed. The methodology involved in the isolation of the fatty acids is described briefly. It seems clear now that most of these acids are made by ehain elongation of intermediate length fatty acids by addition of acetate residues. The unsaturated acids are made by desaturation of the intermediate length acids (palmitic, heptadecanoie, stearic) followed by chain elongation. The hydroxy acids are made directly from the corresponding nonhydroxy acids, saturated, unsaturated, and odd-numbered. All the hydroxy acids undergo oxidative decarboxylation to yield fatty acids containing one less carbon atom. The oddnumbered acids are also made from propionate, which is elongated to intermediate length acids and then to longer acids. The major intermediate length "primer" acid seems to be palmitate, but there is evidence that the stearate used for cerebroside synthesis is also made de novo from acetate. The ganglioside fatty acids were found to turn over somewhat faster than the other fatty acids. Two metabolic pools for the cerebroside acids were found, one with a very high turnover rate, the other with a very low turnover rate.
\end{abstract}

\section{INTRODUCTION}

$\mathrm{T}$ HE BRAIN GLYCOLIPIDS covered in this paper are the cerebrosides, sulfatides, and gangliosides. More specifically, these are ceramide galactoside, cerebroside sulfate (sulfuric acid ester of cerebrosides, attached to the 3-position of the galactose), and the ceramide polysaccharides containing neuraminic acid. These lipids occur as families, differing within each family as to the nature of the fatty acid in the ceramide residue. The cerebrosides

1 Presented at the Prof. Ernst Klenk Symposium on Glycolipids and the Nervous System, AOCS meeting, Houston, April 1965.

Abbreviations: RCA, relative carboxyl activity (activity in $\mathrm{COOH}$ group $\times 100 /$ activity in total FA) ; FA, fatty acid, HFA, 2-hydroxy fatty acid; NFA, nonhydroxy fatty acid; 18:0, stearic acid; 18:1, oleic acid; $16: 1^{9}$, palmitoleic acid as is 16:1w7. An h symbolizes a 2-hydroxy $\mathrm{FA}$, thus $\mathrm{h} 24: 0$ is cerebronic acid (2-hydroxylignoceric acid). A $k$ indicates a 2-keto $\mathrm{FA}$. $\mathrm{O}$ is chloroform; $\mathrm{E}$, ether; $\mathrm{H}$, hexane; $M$, methanol. and sulfatides contain NFA and HFA, saturated and unsaturated; the gangliosides, however, contain only NFA in which there are only traces of unsaturated acids. In the cerebrosides and sulfatides there are two elusters of FA: those around 18 earbons long and those around 24 carbons long. In the gangliosides there is only one cluster, centering around 18:0, with negligible amounts of 22:0 and 24:0.

Other points of contrast between gangliosides and the other two can be made: the former occurs primarily in brain gray matter, the latter are primarily in white. The former has glucose attached to the ceramide residue, the latter have galactose. The former has only traces of odd-numbered FA; the latter can contain considerable amounts of $\mathrm{C}_{23}$ and $\mathrm{C}_{25}$ FA. Further differences, particularly in the metabolism of the two groups of glycolipids, will be deseribed below.

This paper describes mainly the studies carried out in this laboratory, generally with live rats. It discusses the special problems of isolation that were encountered, as well as the interpretation of the isotopic data that were obtained. It is hoped that the approaches user will be of value in other types of studies.

\section{SPECIAL PROBLEMS OF ISOLATION}

Technical problems made this study particularly difficult. These arose from several factors:

(a) Judging by experiments with adult animals the blood-brain barrier causes the brain to come out second-best when there is a competition for intraperitoneally administered isotopic precursors. The liver and other organs pick up most of the injected material, so that large amounts of radioactivity must be administered to each animal in order to get useful levels of incorporation into the brain. While intracranial injection yields much better utilization of labeled material, it does not seem to be reproducible or physiological enough to warrant use in turnover and other quantitative studies. Because of the blood-brain barrier and the relatively leisurely rate of metabolism in the brain, the glycolipid FA obtained from labeled acetate administration have low specific activities. This prevents use of the convenient GLC-ionization chamber combinations, which allow rapid determination of the radioactivity in each FA. 
(b) If the individual FA in each glycolipid family is to be quantified and its relative radioactivity is to be measured, the glycolipid family must be isolated from the brain in nearly $100 \%$ yield. Methods which involve losses inevitably give unequal losses of the various individual members.

(c) Because of the high amounts of odd FA in many glycolipid samples, GLC separations of the highest quality are needed.

(d) Since GLC methods, on a preparative seale at least, do not give complete separation of saturated from unsaturated FA, and NFA from HFA, it is necessary to carry out preliminary class separations.

(e) The methyl esters of the 2-hydroxy FA do not, in our hands, undergo GLC separation without destruction. It is therefore necessary to protect the $\mathrm{OH}$ group prior to GLC. In experiments where the isolated $\mathrm{HFA}$ is to be degraded chemically, it is necessary to use an easily removed protecting group.

(f) Because of the great length of the glycolipid FA (up to $\mathrm{C}_{23}$ ), particularly stringent requirements are placed on the techniques for GLC. "Bleed" materials from packings contaminate the GLC effluents.

This list of difficulties is offered to enlist not only the reader's sympathy but also his skepticism toward studies in which the importance of the factors was inadequately appreciated.

\section{SPECIFIC METHODS OF ISOLATION}

\section{Ganglioside F'atty Acids}

As in most of the recent methods for ganglioside isolation or analysis, we use a version of the later extraction-partitioning system of Folch, Lees, and Sloane Stanley $(8,26,17)$. The upper layer of this solvent system contains nearly all the gangliosides, as well as an unknown lipid in which the FA are ester-linked. Isolation procedures which rely on dialysis as the primary purification step (following partition) cannot eliminate this impurity. The ester-linked FA account for nearly all the $18: 1$ in such extracts.

For simple analytical work, we remove most of the esterified FA by a back-extraction with "Folch lower phase" (8), but for isotopic work we saponify the ganglioside extract with mild aqueous alkali, extract the free FA, and then process the purified gangliosides. The FA are obtained by evaporating the solution to dryness, methanolyzing with HCl-M, and extracting with hexane. We have made the methanolysis step convenient and reliable by using a test tube with an O-ring closure (25).

\section{Cerebrosides and Sulfatides}

In our earlier work $(36,18)$ we used Florisil to remove nonpolar lipids (mainly cholesterol), gangliosides, and nearly all the phosphatides from the total brain lipids. The potentialities of this interesting and economical adsorbent were thus brought to the attention of workers in the field of the complex lipids. Following the suggestion of Carroll (5) we have more recently been using Florisil deactivated by addition of water. We first dry the powder thoroughly by heating at $600 \mathrm{C}$ for $60 \mathrm{~min}$, then add $8 \mathrm{ml}$ water per $100 \mathrm{~g}$ powder. The separating power of such Florisil undergoes a rather sudden deterioration after 3 months, and we discard the outdated material. It is likely that cold storage of the wetted material would prolong its life. Perhaps the adsorbent, which is coprecipitated silica gel and magnesia, slowly reacts in the presence of water to form magnesium silicate.

Sulfatides are not readily separated from cerebrosides by Florisil, so we used synthetic ion exchange resins to isolate the sulfatides from the mixture (37). Impurities in the resins, as well as other exchangers we tried, kept us from preparing pure sulfatides by this approach. As Rouser and his co-workers have shown (39), it is possible to make ion exchange practical by purifying the ion exchanger and eluting reagent very extensively.

Our previous methods called for the use of large Florisil columns since much of the total brain lipids had to be retained by the adsorbent. We now carry out a preliminary eleavage of the ester-linked lipids, following removal of gangliosides by solvent partitioning (17). The lipids are stirred $1 \mathrm{hr}$ at room temperature in $0.07 \mathrm{~N} \mathrm{NaOH}$ in C-M $2: 1$, then partitioned with aqueous acetic acid to remove glycerophosphate esters. This treatment converts the ester-linked FA and part of the free FA (7) to methyl esters, which are then readily isolated by Florisil chromatography. The same column also yields brain cholesterol quantitatively. Cerebrosides and sulfatides are eluted with C-M 3:1. Because of the prior methanolysis step, the Florisil column has to adsorb just the cholesterol, cerebrosides, sulfatides, and lyso derivatives of the alkenyl and alkyl phosphatides. If the cholesterol is not wanted, it can be eluted with the methyl esters, thereby reducing the size of the column further.

This procedure is of additional interest as it yields the ester-linked $\mathrm{FA}$ in the form of 
methyl esters, ready for GLC, by a very mild and rapid process. There is no contamination by acetals or aldehydes sinee the lysophosphatidal derivatives formed during transesterification are held back by the Florisil. Most of the free $\mathbf{F A}$ are also held by the Florisil under these conditions.

The availability of a superior quality of siliea gel (Unisil, Clarkson Chemical Co.) has made it possible to get good separation of the two glycolipids obtained from Florisil columns (17). The mixture is applied to the Unisil column in C-M $98: 2$, and eluted further with this solvent to remove ceramide. Elution with $\mathrm{C}-\mathrm{M}$ 94:6 gives the cerebrosides, and C-M 85:15 gives the sulfatides, slightly contaminated with cerebroside. This method is the simplest one available.

\section{Isolation and Analysis of Cerebroside Fatty Acids}

The methyl esters of the NFA and HFA can be separated readily with Florisil columns $(18,20)$. The unsaturated and saturated esters in each group can be separated with Florisil by first forming the methoxy mercuriacetate derivative of the former esters. The mercury derivative is sufficiently polar that it sticks firmly to Florisil. The saturated esters are eluted with hexane or $\mathrm{H}-\mathrm{E}$, and the mercury derivative is eluted with chloroform-alcohol$\mathrm{HCl}$. The $\mathrm{HCl}$ regenerates the original unsaturated ester during elution. Now that good silica gel is available the above separations can be performed equally well with that adsorbent; however, Florisil is somewhat cheaper.

In the course of various problems we have also isolated the hydroxy acids as the copper chelate $(20)$, and have separated the saturated from unsaturated $\mathrm{FA}$ esters with silver nitratesilica gel columns (21). The OH group does not interfere in the latter method, but there is appreciable overlap in the elution of unsaturated NFA and saturated HFA.

The HFA methyl esters can under some conditions apparently be isolated by GLC without derivatizing the $\mathrm{OH}$ group (34). However, we found it necessary to form the methyl ethers (18) or acetate esters $(11,20)$. The acetate esters are more convenient to make and are necessary when subsequent degradations are to be performed, but the methyl ethers separate more sharply and at lower temperatures in GLC.

Application of these techniques led initially to the diseovery that the NFA and HFA in rat and human cerebrosides greatly resemble one another in chain length distribution, except for the relative absence of h18:0 $(18,35)$. The saturated FA of both types differ in distribution from the unsaturated FA. On this basis we suggested that the NFA are made from the HFA or vice versa. A second major finding was the high concentrations of the $\mathrm{C}_{2 \mathrm{~s}}$ and $\mathrm{C}_{25} \mathrm{FA}$, the relative amounts inereasing with age remarkably (19). As is now known, this is the result of conversion of the $\mathrm{C}_{24}$ and $\mathrm{C}_{26}$ acids to the odd-numbered $\mathrm{FA}$ by a 1-carbon degradation system.

\section{CHAIN ELONGATION vS DE NOVO SYNTHESIS OF THE GLYCOLIPID FATTY ACIDS}

\section{Interpretation of Isotopic Data}

It is now well established by in vitro work that there are two main pathways of biosynthesis of NFA. One is the de novo route, the other is chain elongation of intermediate length FA $(13,15)$. The formex system has been prepared in soluble form from various organisms and tissues, including brain (2). This system is able to synthesize 16:0 from acetyl $\mathrm{Co} \Lambda$, via malonyl CoA, in one fell swoop without appreciable dilution by adjacent short-chain FA molecules. (The intermediate formation of malonate, incidentally, was first proposed to a scientifie gathering at a Gordon Research Conference in 1958 by the senior author.) There is evidence that 14:0 is also formed by the soluble system, but it is not yet clear whether any $18: 0$ is formed in this way.

An enzyme system which elongates $\mathrm{FA}$ is found in mitochondria and acts by adding $\mathrm{C}_{2}$ units (acetyl CoA or malonyl $\mathrm{CoA}$ ) to medium length acids, such as $16: 0$ or $16: 1$. Microsomes are known to synthesize FA but the mechanism is not yet known. Since somewhat more formation of 18:0 than $16: 0$ is observed in microsomes (28), one might expect that both systems are present. However, on the basis of findings described below, we suggest that the microsomes make both acids by the de novo route.

A way of determining which route is operating in a live animal is to administer COOH-labeled acetate, isolate the $\mathbf{F A}$ of interest, and decarboxylate it. If the FA is made only by the de novo system, the radioactivity in each odd carbon atom should be the same, regardless of the time at which the animal is sacrificed. Thus the activity in the FA's carboxyl carbon should be $1 / 8$ the total activity in the case of $16: 0,1 / 9$ the total in 
18:0, ete. On a percentage basis, the RCA for $16: 0$ should be $12.5 \%$.

If a FA is made by chain elongation, the RCA can be greater than, equal to, or less than the de novo value, depending on when the animal is killed after the isotope has been administered. This can be seen by considering the relative activities in the acetate and palmitate pools. Shortly after injection of acetate1- $\mathrm{C}^{14}$, the brain acetate pool becomes highly radioactive. The 16:0 pool in brain is assumed to consist of free palmitic acid, previously formed by synthesis from cold acetate as well as breakdown of complex lipids. Each nonradioactive 16:0 molecule condenses with a labeled acetate residue, forming 18:0 in which all of the $\mathrm{C}^{14}$ is in the $\mathrm{COOH}$ group $(\mathrm{RCA}=$ 100\%). As time passes, the 16:0 pool becomes highly radioactive and the acetate pool loses much of its activity due to the rapid metabolism characteristie of this intermediate. Thus 18:0 molecules made later will contain rather little activity in their $\mathrm{COOH}$ groups, the $\mathrm{RCA}$ thus falling below the de novo value.

Samples of 18:0 isolated at various time intervals will exhibit a wide range of RCA's, depending on the relative turnover rates of acetate, 16:0, and 18:0. At all times the isolated 18:0 will consist of a mixture of molecules formed at different times and with differing RCA's. If one were to carry out such a study with only a single time point, one might accidentally find an RCA characteristic of the de novo value.

As was indicated above, it is conceivable that the acetate pool might be used to form 16:0 de novo and then, without delay, used to elongate the 16:0 molecules. In this case it would appear that 18:0 was made de novo. However, it is known that free $\mathrm{FA}$, including $16: 0$, occur in brain $(7,40)$ and that many of the complex lipid molecules of brain undergo hydrolytic release of their FA moieties, pre-
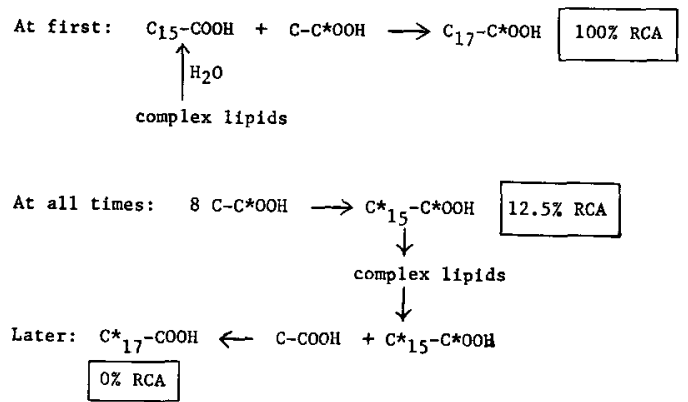

FIG. 1. Sequence of events in brain following intraperitoneal injection of acetate-1- $\mathrm{C}^{\mathrm{14}}$. sumably feeding FA into the free FA pool. Moreover, recent experiments with microsomal FA synthesis indicate that the newly synthesized FA are immediately incorporated into complex lipids, rather than released as free FA. One might therefore expect that the chain elongation enzymes would have available preformed, nonradioactive 16:0. The above hypothesis is summarized in Figure 1.

We have obtained the expected data in the ease of $16: 0$ and 18:0 of the total brain lipids (11). Here, the RCA of 16:0 ranged between 12.2 and 12.9 during a 56 day time period, during which time the specific activity dropped 31-fold. (The theoretical value $=1 / 8=12.5 \%$.) Meantime the 18:0 RCA dropped from 20.7 at $4 \mathrm{hr}$ to 6.0 at 56 days (de novo value = $11.1 \%$ ). Evidently all or most of the 16:0 and 18:0 fit the pattern predieted above.

\section{Relative Carboxyl Activities of Ganglioside Stearate}

Three groups of young rats were injected with acetate-1- $\mathrm{C}^{14}$, then killed in subgroups of five at various time intervals (see Fig. 2). The three groups differed in their initial ages: 7, 13 , and 22 days, chosen to represent the beginning of myelination, the time of most active myelination, and the point of slowing myelination. The brains of all five rats in each time point were pooled and the 18:0 was isolated from the gangliosides and decarboxylated (17). Figure 2 shows the RCA's obtained at each time point. It is evident that ganglioside 18:0 follows the predicted pattern of chain elongation from 16:0 and that there

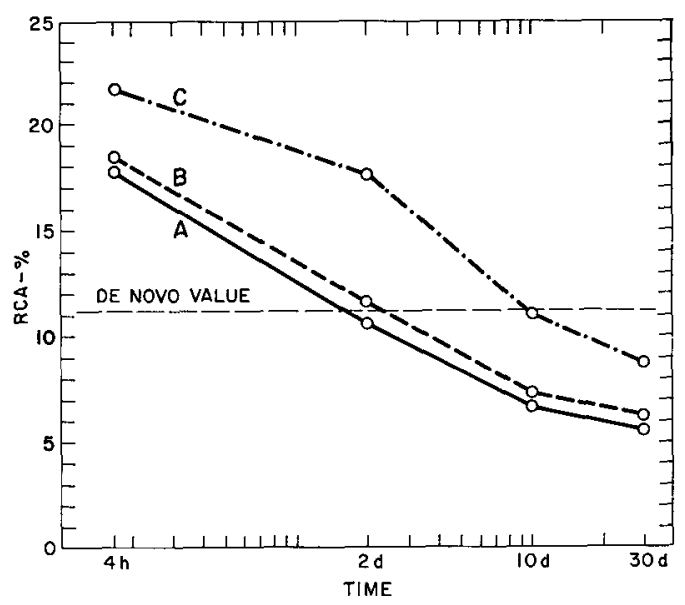

FIG. 2. Relative radioactivity in carboxyl carbon of ganglioside stearate as a function of age. $A$, rats injected at 7 days of age; $B$, rats injected at 13 days; $C$, rats injected at 22 days. 
is no qualitative difference in mode of synthesis in the three age groups.

The older animals show higher RCA's at each time point, probably because the turnover rates of the FA are slower in the older animals.

\section{Relative Carboxyl Activities of the Cerebroside Nonhydroxy Fatty Acids}

Table I shows the results of two studies in which rats were given acetate-1- $\mathrm{C}^{14}$ and the cerebroside lignocerate $(24: 0)$ was isolated and decarboxylated. The de novo value for lignocerate $\mathrm{RCA}$ is $1 / 12=8.3 \%$. It can be seen that high RCA's were found in the early time points, as with ganglioside and total brain 18:0, and the RCA's declined with time. However, only the youngest group of rats showed RCA's below the de novo value, evidently because of a considerable decrease in turnover rate with increasing age. It is evident that cerebroside $24: 0$ is made by chain elongation.

Fuleo and Mead carried out a similar study (9), killing at only one time point, and found an RCA for 24:0 of $10.3 \%$. Since this is moderately close to the de novo value of $8.3 \%$ they concluded that $24: 0$ is made directly from acetate, with little preliminary formation of shorter acids. In one of our experiments with rats of the same age (line 1 , Table I), we found a similar value after two days $(9.4 \%)$, but it is evident that this is only a coincidence of timing.

We also examined the 18:0 from cerebrosides in the above experiments and here (Table II) the RCA's come rather elose to the de novo value $(11.1 \%)$. Only one point $(18.2 \%)$ indicates the existence of chain lengthening. There is thus good reason to believe that the 18:0 used for cerebroside synthesis is made by a hitherto uncharacterized synthetic system. Sphingosine (3), psychosine (6), and cerebroside (1) are made by microsomes, which are also known to utilize acetate to make labeled complex lipids (28). It is thus quite

\section{TABLE I}

Relative Carboxyl Activity in Biosynthesized Cerebroside Lignocerate following Injection of Acetate-1- $\mathrm{C}^{14}$

\begin{tabular}{|c|c|c|c|c|c|c|c|}
\hline \multirow{2}{*}{$\begin{array}{l}\text { Age of rats } \\
\text { at time of } \\
\text { injection }\end{array}$} & \multicolumn{7}{|c|}{ Time of sacrifice after injection } \\
\hline & $4 \mathrm{hr}$ & $10 \mathrm{hr}$ & $2 d$ & $4 d$ & $10 \mathrm{~d}$ & $28-30 d$ & $56 \mathrm{~d}$ \\
\hline Days & & & & & & & \\
\hline 13 & 13.4 & 一 & 9.4 & - & 6.5 & 5.5 & $\cdots$ \\
\hline 22 & 18.2 & 一 & 13.6 & 一 & 12.1 & 11.2 & 一 \\
\hline 23 & 15.7 & 15.6 & - & 13.1 & - & 8.1 & 7.8 \\
\hline
\end{tabular}

possible that these particles make 18:0 de novo and elongate it to form $20: 0,22: 0$, etc. Liver microsomes can convert malonyl-CoA to FA, mainly to $18: 0$ rather than $16: 0(28)$, and it would be of interest to isolate these particles after in vivo administration of labeled acetate to see if the microsomal lipids, freshly formed, contain uniformly labeled 18:0.

\section{Direct Evidence for Chain Lengthening in Sphingolipids}

Additional evidence for the ehain elongation system was obtained by injeeting emulsions of palmitate-1- $\mathrm{C}^{14}$ and stearate-1- $\mathrm{C}^{14}$ directly into the brains of 22-day-old rats (12). The major sphingolipids (cerebrosides + sulfatides + sphingomyelin) were isolated from the brains $24 \mathrm{hr}$ later and their FA were decarboxylated. The 16:0 isolated after palmitate- $\mathrm{C}^{14}$ injection contained nearly all its radioactivity in the original position (96\% RCA), showing that degradation to acetate and resynthesis of 16:0 $(12.5 \% \mathrm{RCA})$ was a minor route, and that part of the injected FA was incorporated into the sphingolipids. Similarly, the 18:0 isolated after stearate- $\mathrm{C}^{14}$ injection was incorporated very directly, too $(97 \% \mathrm{RCA})$.

The 18:0 isolated after palmitate- $\mathrm{C}^{14}$ injection was rather highly labeled and its $\mathrm{RCA}$ was $3 \%$. Evidently the injected 16:0 was elongated with acetate that was relatively nonradioactive. The 16:0 isolated after stearate$\mathrm{C}^{14}$ injection was not highly labeled, evidently because it was made purely by $\beta$-oxidation of 18:0 to acetate, followed by de novo formation. The RCA for this 16:0 was 15\%, a little too high for the de novo value $(12.5 \%)$; it is likely that a small amount of highly labeled 18:0 (97\% RCA) contaminated the 16:0 during GLC.

Radioactivity was also found in 22:0 and 24:0 of the sphingolipids and here the RCA's were $3-5 \%$ with both groups of injected rats. This is direct evidence showing that both $16: 0$ and 18:0 ean be elongated to $22: 0$ and $24: 0$, the elongation having taken place with acetate of relatively low specifie activity.

\section{TABLE II}

Relative Carboxyl Activity in Cerebroside Stearate following Intraperitoneal Injection of Acetate-1-C ${ }^{14}$

\begin{tabular}{|c|c|c|c|c|c|c|c|}
\hline \multirow{2}{*}{$\begin{array}{l}\text { Age of rats } \\
\text { at time of } \\
\text { injection }\end{array}$} & \multicolumn{7}{|c|}{ Time of sacrifice after injection } \\
\hline & $4 h r$ & $10 \mathrm{hr}$ & $2 \mathrm{~d}$ & $4 \mathrm{~d}$ & $10 d$ & $28-30$ & d $56 d$ \\
\hline Days & & & & & & & \\
\hline 13 & 11.7 & $\longrightarrow$ & 9.8 & - & 10.8 & 9.4 & 一 \\
\hline 23 & 18.2 & 10.9 & - & 11.2 & $\longrightarrow$ & 10.9 & 10.2 \\
\hline
\end{tabular}




\section{Formation of the Odd-Numbered Fatty Acids}

The major odd-numbered NFA in the glyeolipids are 23:0 and $25: 1$, with smaller amounts of $23: 1$ and $25: 0$. Small amounts of $15: 0$ and 17:0 occur in brain and elsewhere, and the results of in vitro work with those acids indicate that they are made by the same de novo system that makes 16:0 (4). The only difference is that the starting substrate is propionyl-CoA instead of acetyl-CoA. To see whether a similar system exists in brain, we injected propionate-1- $\mathrm{C}^{14}$ intraperitoneally into a group of rats, then isolated the saturated NFA from the cerebrosides (10).

The data showed that the odd-numbered acids exhibited the highest specific activities by far. Similar results were obtained when the total brain FA were isolated, in which case 15:0 and 17:0 had much more activity. In view of the previously described data for the even-numbered acids, it seems very likely that 15:0 and 17:0 are made from propionate + acetate (malonate) and that these are elongated with $\mathrm{C}_{2}$ residues to form the longer oddnumbered FA so characteristic of the glycolipids.

Evidence for a second route of biosynthesis is presented later.

\section{Formation of the Unsaturated Sphingolipid Acids}

Surprisingly little is known from in vitro work about the mode of formation of the unsaturated acids. For many years the basic fact was known that 18:0 can be desaturated to form $18: 1^{8}$ and it appeared likely that $16: 0$ is desaturated similarly, to form $16: 1^{9}$ (palmitoleate). Presumably the same enzyme aets on both substrates, removing two hydrogen atoms in both cases at the 9,10 position (counting from the $\mathrm{COOH}$ end). In the case of the longer unsaturated NFA of the glycolipids, one would expect this enzyme to form $24: 1^{8}$, $26: 1^{9}$, etc., from the saturated analogs. However, the only isomers of known structure at the time we began this study were $24: 1^{15}$ (nervonate), $22: 1^{13}$, and $20: 1^{11}$. From this observation it would appear unlikely that desaturation of long FA takes place.

Examination of the sphingolipid unsaturated FA from a different viewpoint-from the methyl or $\omega$ end-places the acids in a more coherent system. If we locate the double bonds by counting from the $\omega$ end, we find that they are all in the oleate family: $18: 1 \omega 9,20: 1 \omega 9$, $22: 1 \omega 9$, and $24: 1 \omega 9$. It is then reasonable to assume that the chain elongation system used by the brain to make the glycolipid acids acts similarly on oleate.

Fulco and Mead, in the same labeled acetate experiment mentioned above (9), isolated nervonate and decarboxylated it. Its RCA was $20.2 \%$, much different from the $10.3 \%$ they found for the saturated analog. Since they had concluded that the latter is formed by de novo synthesis, they then concluded that $24: 1$ is formed by the alternative method, ehain elongation. Noting the above-mentioned similarity in double bond position, they suggested oleate is the precursor of nervonate.

We sought to make a direct demonstration of the conversion by injecting oleate-1- $\mathrm{C}^{14}$ directly into rat brains, as in the labeled palmitate and stearate experiments. The results were somewhat different (22), for the long unsaturated FA showed very high RCA's: $74 \%$ for $24: 1,53 \%$ for $22: 1$, and $86 \%$ for $20: 1$. An additional surprise is that relatively little of the injected oleate was incorporated into the sphingolipids. Part of the explanation for the latter finding is that 18:1 is a very minor acid in this group of complex lipids, and it apparently turns over very slowly (if at all).

It would appear from the above results that very little of the injected oleate entered the cells making nervonate, etc., and much of the $18: 1$ was simply oxidized to acetate- $1-\mathrm{C}^{14}$. The acetate did enter the synthesizing cells and was used to elongate the 18:1 already present in the cells. However, if labeled acetate were used to elongate only 18:1, the resultant 24:1 would have $1 / 3$ of its $\mathrm{C}^{14}$ in its $\mathrm{COOH}$ group (since three $\mathrm{C}_{2}$ units are needed). Since the RCA for 24:1 was far above $33 \%$, it must be made in part by elongation of $22: 1$ and $20: 1$. Evidently the sphingolipid-synthesizing cells contain pools of $22: 1$ and $20: 1$, as well as 18:1, all of which can be used for ehain elongation. It is likely that similar pools of saturated FA are also present.

\section{Evidence from Ozonolysis of the Sphingolipid Acids}

Palmitoleate, $16: 1 \omega 7$, exists in brain, albeit in much lower concentrations than oleate, and it too should be subject to ehain elongation if the elongating enzymes are not too specific. 17:1 also exists and should give rise to the odd-numbered unsaturated FA, none of whose structures was known. In order to confirm these guesses, we processed pig brains to isolate the sphingolipids and their individual unsaturated FA. GLC yielded these acids as single peaks which showed the expeeted in- 
frared absorption spectra for cis-FA. A microozonolysis procedure was devised (21) which permitted quantitative determination of the fragments of each FA isomer, starting with $1.5-3 \mathrm{mg}$ of sample. The results of analyzing the mono- and diunsaturated FA are shown in Table III, which gives the abundance of each isomer, classified according to its $\omega$-family. Similar data are shown for the HFA from the same brains (23).

The number of isomers found within each chain length was unexpectedly large; most of these had previously not been reported in nature. The existenoe of even- and oddnumbered diunsaturated FA in the sphingolipids is also of interest.

The $\omega 6$ family occurs only in the dienes and is evidently due to chain elongation of linoleate. The $\omega 7$ family is found in all of the acids and presumably arises from palmitoleate. Elongation of $16: 1 \omega 7$ should give rise to $18: 1 \omega 7$ (cis-vaccenic acid) as well as the longer members of this family. Cis-vaceenic acid does indeed exist in brain, as we found in a later study (24) of the ester-linked FA of pig brain. While this acid had been known to occur in animals for some time, it was considered a rare acid. However, we found this to be the second most common monounsaturated FA, second only to oleate and much more abundant than palmitoleate. As was shown very recently in vitro (15), eis-vaccenate is formed by elongation of palmitoleate, confirming our suggestion that the latter is elongated to form a series of $\omega 7 \mathrm{FA}$.

The odd-numbered acids of the $\omega 7$ family may be presumed to originate from the evennumbered members by 1-earbon degradation according to the hydroxylation-oxidation system described in a later section of this paper.

The occurrence of the $\omega 8$ family only in the odd-numbered FA must mean that these acids are formed by elongation of a primary acid, $17: 1 \omega 8$. This acid is presumably formed by desaturation of $17: 0$ at the 9,10 position.

The oleate, or $\omega 9$ family of acids is like the $\omega 7$ family in that it is represented in all chain lengths and in the dienes. These acids are probably formed by elongation of oleate and the odd-numbered acids are formed by 1-carbon degradation of the longer acids. Thus all of the above unsaturated acids may be considered as arising initially from the desaturating enzyme which acts on the 9,10 position from the $\mathrm{COOH}$ end of $16: 0,17: 0$, and $18: 0$.

The w10 family of Table III can probably be explained as arising by elongation of $16: 1 \omega 10$. The odd-numbered acids presumably are formed from the longer acids $(26: 1 \omega 10$ and $24: 1 \omega 10)$ by the 1-carbon degradation system. The postulated "primer" acid, $16: 1 \omega 10$, was found in the ester-linked acids (24) as was a long line of longer $\omega 10$ FA. Perhaps another desaturating enzyme exists in brain which is specific for the 6,7 position of $\mathrm{FA}$ (counting from the $\mathrm{COOH}$ end).

The above hypotheses are based on the assumption that the chain elongating enzymes can act on 16-,17-, and 18-carbon FA as their "primer." This is consistent with our previous proposal that 18:0 is made de novo in mierosomes and lengthened to form the cerebroside acids and with the results eited above with intracranial injection of labeled $16: 0$ and 18:0. FA desaturation of 18:0 takes place in microsomes (29).

The role of palmitoleate in chain elongation was demonstrated by ozonolysis of the $24: 1$ isolated in the previously described experiment (22) in which oleate-1- $\mathrm{C}^{1.4}$ was injected into rats intracranially. The $24: 1$ yielded two dicarboxy acids, $\mathrm{C}_{15}$ and $\mathrm{C}_{17}$, and two monocar-

TABLE III

Occurrence of the Unsaturated Fatty Acids of Pig Brain Sphingoipids

\begin{tabular}{|c|c|c|c|c|c|c|c|c|}
\hline \multirow{2}{*}{$\begin{array}{c}\text { Fatty } \\
\text { acid } \\
\text { familya }\end{array}$} & \multicolumn{5}{|c|}{ Monounsaturated fatty acids } & \multicolumn{3}{|c|}{ Diunsaturated acids } \\
\hline & $22: 1$ & $23: 1$ & $24: 1$ & $25: 1$ & $26: 1$ & $24: 2$ & $25: 2$ & $26: 2$ \\
\hline$\omega 6 \quad \mathrm{NFA}$ & - & - & - & $\longrightarrow$ & - & 1.9 & 0.14 & 0.14 \\
\hline$\omega 7$ & 21 & 4,5 & 44 & 3.7 & 13 & 0.3 & 0.07 & 0.22 \\
\hline$\omega 8$ & - & 2.7 & - & 1.3 & - & - & - & - \\
\hline$\omega 9$ & 3.7 & 1.5 & 170 & 10 & 10 & 0.4 & 0.06 & 0.13 \\
\hline$\omega 10 "$ & 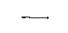 & 0.1 & 4.4 & 1.3 & 2.9 & 一 & 0.01 & 0.02 \\
\hline$\omega 7$ HFA & 3.2 & 1.6 & 33 & 3.8 & 18 & & \multirow{4}{*}{\multicolumn{2}{|c|}{ isolated }} \\
\hline$\omega 8$ & - & 0.7 & - & 0.7 & - & & & \\
\hline$\omega 9$ & 0.5 & 0.4 & 44 & 2.8 & 7.3 & & & \\
\hline$\omega 10 \quad "$ & 0.1 & 0.03 & 3.2 & 0.2 & 1.0 & & & \\
\hline
\end{tabular}

a Position of double bond counting from the methyl end. In the case of the diunsaturated FA, it indicates the position of the double bond closer to the methyl end.

Data are in mg FA/100 g fresh brain. 
boxy acids, $7: 0$ and $9: 0$. The distribution of activity in the original $24: 1$ isomers was as follows:

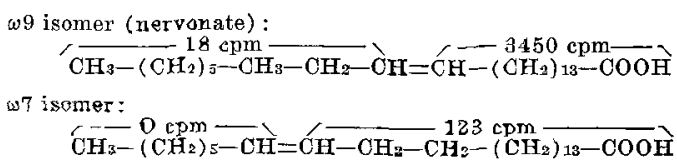

These data are consistent with the idea that the injected oleate was primarily oxidized to acetate, which was then used to elongate nonradioactive palmitoleate and oleate.

\section{FORMATION OF THE 2-FYDROXY FATTY ACIDS}

\section{Studies with Labeled Acetate}

Fulco and Mead, in the same experiment mentioned (9), carried out the first examination of cerebronic acid (h24:0). They injeeted acetate-1- $\mathrm{C}^{1 *}$ intraperitoneally into 13-day-old rats and isolated brain h24:0 (as well as 24:0). Decarboxylation of the HFA yielded a RCA of $10.3 \%$, identical to that of the $24: 0$. Indeed the $\mathrm{C}^{14}$ distribution in the first 3 carbon atoms of each acid showed very similar patterns. From these relations, and the finding that 24:0 had 16 times the specific activity of h24:0, they concluded that the HFA was probably made from the analogous NFA.

The force of the conclusion was weakened by the mode chosen for administering the isotopic acetate. The acetate was injected in 4 daily doses and the animals were killed 3.5 $\mathrm{hr}$ after the last dose. Reiner (38) has pointed out the difficulties that ensue from such a dosage plan, but the mathematical treatment of the problem was a formidable one. A simpler approach ean be made by considering what would happen if one were to give an animal acetate- $-\mathrm{C}^{\mathrm{1}}{ }^{14}$ for several months during its early life. It seems very likely that each FA molecule in the animal would become uniformly labeled (in the odd-numbered positions) and would reveal a $\mathrm{RCA}$ corresponding to the theoretical de novo value, even if the acid had been made by chain elongation. Lignocerate, for example, is made from 16:0 or $18: 0$ and acetate, all of which would haye the same specific activity.

In the more typical 1-pulse type of isotope experiment, the molecules of $24: 0$ tormed at first are made from highly labeled acetate and relatively unlabeled $16: 0$ or 18:0. A second pulse of labeled acetate would find itself adding on to $16: 0$ which is now more radioactive. Each successive pulse maintains the specific aetivity of the brain acetate, which is now adiding on to highly and similarly labeled $16: 0$. The Fulco-Mead experiment was concluded shortly after the last injection, while the brain $16: 0$ was still at a high level of activity. Under this condition one would expect to find a RCA for each brain acid (or any other FA made from a slowly metabolizing A) close to its de novo value. Indeed, Fuleo and Mead confirmed this in a later analysis of the 18:0 from these rat brains (31). Here the RCA was $12.7 \%$ for eerebroside $18: 0$ and $11.7 \%$ for ester-linked 18:0 (de novo $=11.1 \%$ ). They concluded from this that all brain 18:0, like $24: 0$, is made from acetate without dilution with inaetive $16: 0$.

The liver 18:0 from the same rats showed a RCA of $30 \%$, which indicates chain elongation must have taken place. Why the difference between brain and liver? FA metabolism is undoubtedly much faster in liver so that the 16:0 formed after the first 3 isotopic injections had already turned over and lost much of its radioactivity by the time the last pulse of acetate was administered. Thus this experiment with respect to liver was much like a typical 1-pulse experiment; during the $3.5 \mathrm{hr}$ following the last injection the labeled acetate was adding on to relatively nonradioactive $16: 0$, produeing 18:0 with a high RCA.

It should be evident from this discussion that the data resulting from a multipulse experiment can be misleading when the animals are sacrificed shortly after the last injection.

As part of our time study of the NFA of brain cerebrosides formed from labeled acetate (11), we also isolated the major saturated HFA. The RCA's of 24:0 and h24:0 were quite similar throughout the period examined, $4 \mathrm{hr}$ to 56 days (see line 3 of Table I for the 24.0 data). This relation is consistent with the idea that 24:0 is the precursor of h24:0 (or vice versa). A probable sequence of events in this case is:

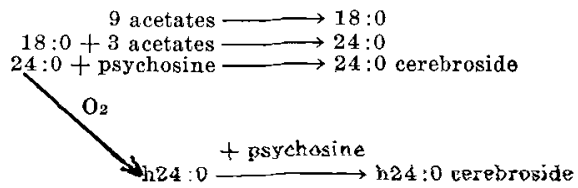

If the 2 cerebrosides, kerasin and cerebron, had somewhat different rates of synthesis and breakdown one would find the RCA's diverging with time. This follows from the fact that the RCA of a group of molecules is the average of the RCA's of the individual molecules. As new molecules of 24:0 and h24:0 are deposited in cerebroside form, they modify 
the average RCA of the previously deposited molecules at similar rates.

\section{Evidence from Intracranial Injection}

As in the previously described experiments utilizing intracranial injection of labeled 16:0 and 18:0, we also injected lignocerate-1-C $\mathrm{C}^{14}$ and cerebronate-1- $\mathrm{C}^{14}$ (12). In the case of the former compound, only the 24:0 and h24:0 of the cerebrosides were found to be highly labeled. (Unsaturated acids were not isolated). The RCA of the isolated h24:0 was $89 \%$, which means that most of the cerebronate was made quite directly from the 24:0, although some must have been made from 24:0 formed endogenously from labeled acetate (itself derived by $\beta$-oxidation of some of the injected $24: 0)$.

The reverse reaction apparently does not go, at least under these conditions. Almost none of the cerebronate radioactivity found its way into the NFA although incorporation of the cerebronate into the sphingolipids was observed.

Since the reaction probably is carried out by the microsomes, which are noted for their ability to hydroxylate substrates, it is likely that the reaction involves a direct hydroxylation in the 2-position. However, there is the possibility that the NFA is first dehydrogenated in the 2,3-position, then hydrated in the "unnatural" direetion.

A preliminary note has appeared on an enzyme system in plants which converts 16:0 to h16:0 and then to $15: 0$ (14).

\section{The Unsaturated Hydroxy Acids}

Of two possible synthetic routes:

$$
\begin{gathered}
\text { unsaturated FA } \stackrel{+\mathrm{O}_{2}}{\longrightarrow} \text { unsaturated HFA } \\
\text { saturated HFA } \stackrel{-2 \mathrm{H}}{\longrightarrow} \text { unsaturated HFA, }
\end{gathered}
$$

it is likely that the first is the actual one. The evidence presented earlier in this paper showed that it is likely that the unsaturated NFA are made by chain elongation of the medium length unsaturated NFA. It is these acids which are probably hydroxylated.

Further evidence comes from examination of the double bond positions in the HFA of brain (23). As described before, pig brains were processed to yield the individual unsaturated HFA. (homogeneous with respect to chain length) and these were then examined by ozonolysis to determine the amounts of each unsaturated isomer. The even - and oddnumbered HFA between h22:1 and h26:1 were studied (Table III). Each acid contained a member of the $\omega 7$ family and the $\omega 9$ family, presumably derived by elongation of palmitoleate and oleate followed by hydroxylation.

Also present, but only in the odd-numbered HFA, were members of the $\omega 8$ family. As explained before, these are probably formed by elongation of $17: 1 \omega 8$.

Trace amounts of the $\omega 10$ family were also found, as in the unsaturated NFA. Again the evidence points to simple hydroxylation of the analogous unsaturated NFA.

There is a marked difference between HFA and NFA in the relative abundances of the $\omega 7$ and $\omega 9$ families. The total amounts of the two families in the HFA are 60 and $55 \mathrm{mg} / 100$ $g$ brain, respectively; in the NFA they are 86 and $195 \mathrm{mg} / 100 \mathrm{~g}$ brain. Perhaps this reflects a tendency of the hydroxylating enzyme to prefer FA having a longer saturated ehain next to the COOH group. This preference is shown also in a comparison of the relative abundances of the NFA and HFA of all the sphingolipid acids of the same pig brains (Table IV). Here we see that the relative amount of HFA rises with increasing length of the $\mathrm{CH}_{2}$ chain next to the $\mathrm{COOH}$ group (26:0 is a minor exception).

\section{THE 1-CARBON DEGRADATION SYSTEM}

\section{Evidence from Degradation Experiments}

In one of the turnover experiments described above (11), in which weanling rats were injected with acetate- $1-C^{14}$, the cerebroside $\mathrm{h} 23: 0$ acid was isolated and decarboxylated. As predicted from the elongation theory, the RCA was high in the early time points and low in the late time points. Indeed, the RCA went to the lowest observed value in this study, $2.9 \%$. From this it would appear that an oddnumbered FA, probably $17: 0$, is formed first

TABLE IV

Relative Abundances of Hydroxy and Nonhydroxy Acias in Pig Brain Sphingolipids

\begin{tabular}{|c|c|c|c|}
\hline \multicolumn{2}{|c|}{ Acid type } & \multirow[t]{2}{*}{$\begin{array}{l}\text { Number of } \mathrm{CH}_{2} \\
\text { groups attached } \\
\text { to COOH group }\end{array}$} & \multirow{2}{*}{$\begin{array}{c}\begin{array}{c}\text { Ratio of } \\
\text { HEA/NFA }\end{array} \\
0.15\end{array}$} \\
\hline 22 & Monoenes & & \\
\hline 23 & $"$ & 15 & 0.31 \\
\hline 24 & $"$ & 14 & 0.35 \\
\hline 25 & $"$ & 17 & 0.88 \\
\hline 26 & $"$ & 18 & 0.97 \\
\hline & Saturateds & 21 & 1.6 \\
\hline 23 & " & 22 & 2.1 \\
\hline 24 & $"$ & 23 & 2.1 \\
\hline 25 & $"$ & 24 & 2.3 \\
\hline 26 & $"$ & 25 & 1.5 \\
\hline
\end{tabular}

a Calculated for the major isomer in the case of the monoenes (in the case of 25:1 the major NFA isomer is $\omega 9$ and the major HFA isomer is $\omega 7$ ). Data are derived from Table III. 
TABLE V

Distribution of Radioactivity in the Individual Carbon Atoms of Cerebroside Acids after Injection of A cetate $\cdot 1 \cdot \mathbf{C}^{14}$

\begin{tabular}{|c|c|c|c|c|c|c|c|}
\hline \multirow{2}{*}{\multicolumn{2}{|c|}{$\begin{array}{l}\begin{array}{l}\text { Age of rat at } \\
\text { the time of }\end{array} \\
\text { Injection Sacrifice }\end{array}$}} & \multirow{2}{*}{$\begin{array}{c}\text { Fatty acid } \\
\text { degraded }\end{array}$} & \multicolumn{2}{|c|}{ Carbon } & \multirow{2}{*}{$-\frac{\text { atom }}{22}$} & \multicolumn{2}{|c|}{ number ${ }^{a}$} \\
\hline & & & 20 & 21 & & 23 & 24 \\
\hline $23 \mathrm{~d}$ & $51 d$ & h23:0 & - & - & 8.5 & 4.3 & - \\
\hline $23 d$ & $79 \mathrm{~d}$ & $\begin{array}{l}\mathrm{h} 22: 0 \\
\mathrm{~h} 23: 0 \\
\mathrm{~h} 24: 0\end{array}$ & - & $\frac{1.4}{-}$ & $\begin{array}{l}9.1 \\
8.3 \\
-\end{array}$ & $\begin{array}{l}- \\
2.9 \\
0.8\end{array}$ & $\overline{8.4}$ \\
\hline $15 \mathrm{~d}$ & $168 \mathrm{~d}$ & $\begin{array}{l}\mathrm{h} 23: 0 \\
\mathrm{~h} 24: 0 \\
23: 0\end{array}$ & $\overline{6.4}$ & $\begin{array}{l}2.8 \\
1.0 \\
1.9\end{array}$ & $\begin{array}{l}5.8 \\
5.3 \\
4.9\end{array}$ & $\begin{array}{l}0.3 \\
0.6 \\
0.8\end{array}$ & $\overline{9.3}$ \\
\hline
\end{tabular}

a Counting from the methyl end of the FA.

Data are in percent of activity in the carbon atom compared with the entire molecule. The lower three lines are from Mead and Levis (32).

and is then elongated with 3 acetate residues to form $23: 0$, which is then hydroxylated. The rapid drop-off in $\mathrm{RCA}$ suggests that this acid has a high turnover rate. However, the total radioactivity per brain in cerebroside h23:0 showed the most prolonged rise with time of all the cerebroside acids, which suggests that this acid undergoes little or no breakdown. Moreover, the deposition in brain of $h 23: 0$ is the most prolonged and striking, which could mean that this acid does not undergo metabolie attack.

The conflicting pieces of evidence were clarified by degrading the $\mathrm{h} 23: 0$ further to learn the relative activity of carbon 2. The data are shown in Table $V$ and include similar degradation data for $h 22: 0$ and h24:0. By looking at lines 1 and 3 , we see that the second carbon atom of h23:0 (analyzed at 2 different time points) has an unexpectedly high amount of $\mathrm{C}^{14}$. If $\mathrm{h} 23: 0$ were made only by elongation with acetate-1-C $\mathrm{C}^{14}$, the $a$-carbon atom should contain virtually no radioactivity. Indeed, analysis of the even-numbered HFA (lines 2 and 4) does show there is little $\mathrm{C}^{14}$ in the a-atoms. (There should be even less, according to the elongation theory.) The most plausible explanation of the unexpected finding is that part of the h23:0 is made by a 1-carbon degradation of $\mathrm{h} 24: 0$. The $\beta$-earbon of $\mathrm{h} 24: 0$, which should have a $\mathrm{C}^{14}$ content similar to that of the $\mathrm{COOH}$ carbon, becomes the $a$-carbon of $h 23: 0$ and the $a$-carbon of $h 24: 0$ becomes the $\mathrm{COOH}$ of $\mathrm{h} 23: 0$.

The relative activities in the individual atoms are shown in Table $\mathrm{V}$ in columns which align the data for identical atoms.

The 1-earbon degradation scheme is shown in Figure 3.

It is very likely that part of the $h 23: 0$ is further degraded to h22:0. This explains the small but appreciable activity in the a-carbon of the latter acid. (An alternative explanation, which must account for part of the activity in the a-atoms of all FA, is that part of the injected acetate is metabolized to labeled $\mathrm{CO}_{2}$, and part of this finds its way to form acetate labeled in the methyl position. Such acetate, during chain elongation, would yield FA containing some $\mathrm{C}^{14}$ in the even-numbered carbon atoms.)

Mead and Levis (32) earried out a similar experiment which is also summarized in Table

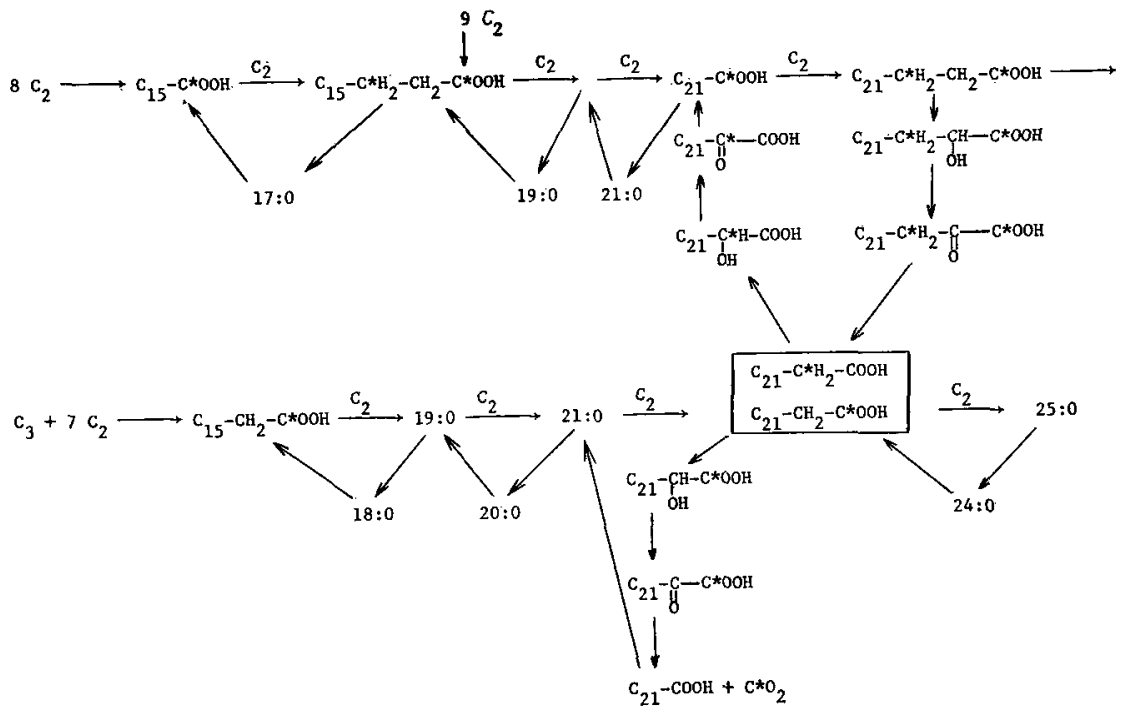

FIG. 3. Formation of the odd-numbered fatty acids by two biosynthetic routes. 
$V$ (last 3 lines). Substantially the same results were found. Incidentally, their RCA for h23:0 was even lower than ours, evidently because the rats were kept for a much longer period before sacrificing.

\section{Evidence from in Vitro Work}

Following this decarboxylation study, Levis and Mead succeeded in demonstrating the 1carbon degradation reaction in vitro (27). They showed that $\mathrm{h} 18: 0$ and $\mathrm{h} 23: 0$ are degraded by rat brain microsomes to form $\mathrm{CO}_{2}$ and the acid with one carbon atom less. ATP and NAD are necessary for the system as well as unknown material found in the cell supernatant. They found that $\mathrm{k} 18: 0$ (2-ketostearate) was also attacked by the enzyme system, somewhat more rapidly than the h18:0. Presumably the hydroxy acid is decarboxylated via the keto acid as intermediate.

At the kind invitation of Dr. Mead, the senior author and Dr. Amiya Hajra spent a month at his laboratory to become familiar with the problem. We have since been studying the enzymatic attack of the brain enzymes on k18:0. Drs. S. S. Parmar and W. E. Davies have also worked in this laboratory on the problem, using an acetone powder of pig brain as the enzyme source and following the reaction by measuring the $\mathrm{C}^{14} \mathrm{O}_{2}$ produced from the keto acid.

It appears that the same cofactors are needed for $\mathrm{k} 18: 0$ and h18:0 oxidation: ATP, NAD, and cell supernatant. Part of the activity in the cell supernatant is removed by Dowex $50-\mathrm{H}^{+}$; this seems to be $\mathrm{Mg}^{++}$. Another part is removed by Dowex 1-OAc-; this can be recovered from the resin by elution with dilute acid. The acidic cofactor is stable to heat in acid but not in alkali and shows the properties of ascorbic acid. Synthetic ascorbic acid does replace the acidic cofactor but is inhibitory at higher concentrations. The optimal concentration in our system is $5 \times 10^{-5} M$, which is unusually low for ascorbic-catalyzed enzyme reactions.

The complete enzyme system, containing ATP, NAD, ascorbate, and $\mathrm{Mg}^{++}$, is seriously inhibited by an iron chelator (o-phenanthroline) or by the more general chelator, EDTA. The EDTA effect is not reversed by washing the particulate enzyme with water unless ferrous chloride is added, so it appears that bound $\mathrm{Fe}^{++}$is also needed for activity.

Incubation under nitrogen drastically lowers activity. Further evidence that the reaction is oxidative was obtained by isolating the non- radioactive product, $17: 0$ acid, by means of TLC and GLC. The enzyme preparation itself contains some free FA (even-numbered), so no carrier was needed. We did add 16:0 aldehyde before isolating the lipoidal products, but reisolation of the aldehyde showed only one peak $(16: 0)$ on GLC.

\section{TURNOVER OF THE GLYCOLIPID FATTY ACIDS}

Turnover data normally consist of measurements of the activity of compounds which are isolated from animals previously injected with a single dose of a radioactive precursor. The animals are sacrificed at various times after the injection and the activities are plotted against time. In order to minimize individual variation among animals and obtain smooth curves, one must sacrifice groups of animals at each time point. Thus such experiments tend to involve a great deal of effort and radioactive material. They are carried out because they yield information that cannot be obtained in other ways.
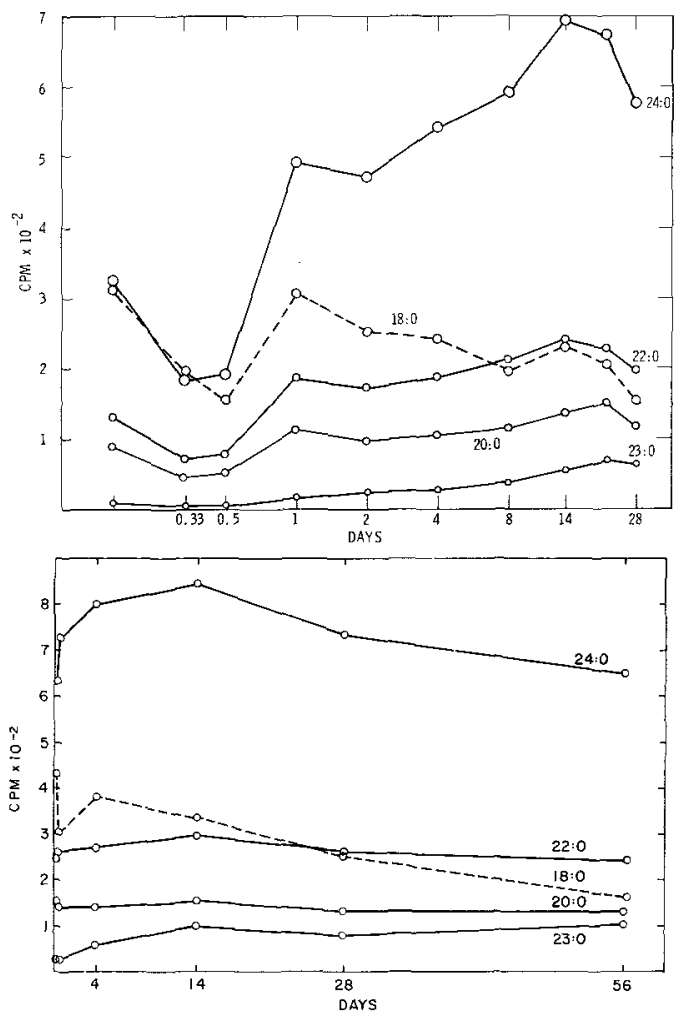

FIG. 4. Total activity per brain in NFA of cerebrosides. Upper figure, rats injected with tritium-labeled acetate. Lower figure, rats injected with COOH-labeled acetate. (From ref. 11, reprinted by permission of the publisher.) 


\section{The Cerebroside Fatty Acids}

Figure 4 shows the results of administering a single dose of acetate- $2-\mathrm{H}^{3}$ (upper curves) or acetate-1- $\mathrm{C}^{14}$ (lower enrves) to male weanling rats, then isolating the cerebroside NFA (11). Let us examine the curves singly, from the top down.

The curve for 24:0 shows a maximal value was reached soon after the injection, at approximately $4 \mathrm{hr}$. (The zero point is not shown because the data are plotted on semilog paper). The activity then declined rapidly, showing rapid metabolism of the cerebrosidebound $24: 0$. Whether the metabolism involved hydrolytic breakdown or, perhaps, sulfation to form sulfatide, we cannot tell from these data. A second rise in activity appears, much more gradual, reaching a peak at roughly 14 days. Again the activity drops off, also much more gradually than the first time. The presence of two separate turnover patterns for one substance must mean that we are observing two pools of $24: 0$ cerebroside (kerasin). The first peak shows there is a pool of kerasin in brain which is formed and destroyed rapidly, with a half-life of roughly one third of a day. The second peak shows there is a pool with a much more leisurely breakdown rate. Since we did not prolong the experiment long enough we must consider the possibility of a third pool which might not, perhaps, undergo any breakdown at all.

The first pool of $24: 0$ is made from 16:0 and/or 18:0 by elongation with highly radioactive acetate. Since the tritium-labeled acetate is known to undergo very rapid oxidation to $\mathrm{T}_{2} \mathrm{O}$ and $\mathrm{CO}_{2}$, the slowly metabolizing 24:0 must be made from some other radioactive precursor. As our decarboxylation and other experiments showed, this radioactive precursor must be labeled 16:0 or 18:0, formed in the initial hours from labeled acetate. The labeled medium-length $F A$ are in large part incorporated into the glycerophosphatides, which show a more rapid metabolism than the cerebrosides (11). As the phosphatides undergo breakdown, the liberated FA are in paxt utilized for chain elongation to form 24:0. Thus the precursor FA pool loses activity only quite slowly and continues to add tritiun over many weeks to the even more slowly netabolizing cerebroside pool.

It is tempting to assign the slowly metabolizing kerasin to myelin and the rapidly metabolizing kerasin to the other structural elements of the brain. Alternatively, the rapidly metabolizing kerasin could be in the gray matter and the other pool in the white. Several workers, including our own group, have shown that cerebroside is not localized only in myelin.

Let us now examine the next curve, for cerebroside 18:0. This FA also shows 2 components, but the second one reaches its maximum fairly soon too. It then shows a gradual decline in activity for the next 27 days or more. The difference between kerasin and 18:0 cerebroside is quite striking with respect to the more slowly metabolizing cerebroside. Perhaps the difference lies in a different subcellular distribution of the two cerebrosides, or in readier attack by enzymes on the less lipoidal 18:0 cerebroside (33).

The next 2 curves, for $22: 0$ and $20: 0$, resemble the 24:0 curve somewhat. The 23:0 curve is atypical, showing a very prolonged rise. The slight dip at the 28-day point may be due to experimental variation. This long period of accumulating isotope indicates that a radioaetive precursor is slowly being made available for 23:0 formation. We now know from the experiments described earlier in this paper that two long-lived precursors are involved: labeled 17:0, formed early from propionate and labeled acetate, and labeled h24:0. Both FA are made available by constant release from glycerophosphatides and cerebrosides.

The lower set of eurves, obtained in the $\mathrm{C}^{14}$ experiment, illustrates an important point of general validity. Note that the very early rise and fall in activity seen in the upper curves is not visible here, except for the cerebroside 18:0. If we had not run the same experiment with tritium-labeled acetate, we would have missed finding the rapidly metabolizing cerebrosides. The explanation for the difference lies in the nature of $\mathrm{F}^{3} \mathrm{~A}$ degradation. When tritium-labeled $16: 0$ or $18: 0$ is degraded by $\beta$-oxidation, most of the tritium is lost as water (and greatly diluted by the body water). The acetate residues formed in $\beta$-degradation are thus only slightly radioactive so that reutilization of these residues will give rise to rather slightly labeled FA. On the other hand, when

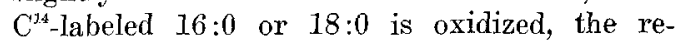
sultant acetate residues still have all their radioactivity. Of course much of this endogenous acetate is metabolized via the Krebs cycle, but still part is reutilized for FA syathesis. The net effect is that the radioactivity in the acetate- $\mathrm{C}^{14}$ pool dies away somewhat more slowly than that in the acetate- $\mathrm{H}^{3}$ pool. This acts to cover up rapid changes in FA pools in the first day after injection. It is very likely 
that other studies of FA metabolism in similar experiments have missed similar disclosures of rapid turnover fractions because of the use of $\mathrm{C}^{14}$

It should be noted also that many turnover studies in brain have neglected the early time points altogether and could not have picked up such fractions anyway.

Returning to the lower part of Figure 4 we see that the curves are similar to the upper part with respect to the slow turnover fraction. This experiment covered a somewhat longer time period (and is plotted arithmetically), so the long period of decline in activity is better delineated. The slowness in deeline of $24: 0$ activity hints at the presence of a nonmetabolizing fraction. Again we see that 18:0 cerebroside oceupies a distinetly different position in the metabolic scheme, as does the 23:0 cerebroside.

Our analytical data from GLC also show the atypical nature of $23: 0$ and $h 23: 0$ cerebrosides, both of which accumulate markedly with age. Evidently the 1-carbon degradation system becomes a prominent system in older animals, in which the synthetic enzymes seem to slow down.

The turnover curves for the HFA of cerebrosides (not shown here) are qualitatively similar to those of Figure 4 except that their second activity peak (with acetate- $\mathrm{H}^{3}$ ) comes somewhat later. This is evidently due to their being formed from the corresponding NFA.

In a later study with tritium-labeled acetate we confirmed the existence of the rapidly metabolizing fraction and found the first activity maximum to be eloser to $2 \mathrm{hr}$ than 4 . This fraction exists also in somewhat older rats. Incidentally, the sphingosine in cerebroside was also found to undergo turnover, so it is apparent that cerebroside molecules undergo complete breakdown or are used in toto in the course of their metabolism.

We also made a turnover study with acetate1-C $\mathrm{C}^{14}$ using rats of three different ages, as described in Figure 2. The rats were killed $4 \mathrm{hr}, 2$ days, 10 days, and 30 days after injection and the activity incorporated into the cerebroside acids was measured (17). The highest activity was found in rats injected at 13 days of age, when myelination is fastest. Some qualitative differences between the 3 age groups were seen. A striking difference was noted in the 18:0 from the youngest rat group which did not show the rapid decline seen in the older rat groups. Moreover, the activity found in 18:0 of the youngest rats was higher than that found in 24:0 (in con- trast to the reverse situation in the older rats). Evidently very young rats make more 18:0 cerebroside than 24:0 cerebroside. This finding is in agreement with that of O'Brien, who found 18:0 cerebroside to be somewhat more plentiful in early childhood (33). The same is true for the FA of human brain sphingomyelin (41).

Another interesting observation was that the data from the rats injected at 7 days revealed the existence of the rapidly metabolizing cerebroside fraction even though the isotope used here was $\mathrm{C}^{14}$. This observation is consistent with the suggestion that the slowly metabolizing fraction is myelin-bound. Myelin formation in rat brain appears to be insignificant until about 9 days of age (16), so during the 2-day period following the $\mathrm{C}^{14}$ injection there must have been incorporation only into the labile cerebrosides and none into the myelin cerebrosides.

\section{The Sulfatide Fatty Acids}

The 18:0 and 24:0 of the sulfatide FA were isolated in the experiment deseribed just above. As with the cerebrosides, the 24:0 activity curve showed a steady rise with time. Surprisingly, the 18:0 eurve also showed a similar steady rise; indeed, in the 7-day and 13-day rat groups the 18:0 rose even more rapidly than the 24:0. In the cerebrosides, the 18:0 activity declined (except in the early period) so the difference is quite marked. The difference may indicate a relatively rapid conversion of 18:0 cerebroside to 18:0 sulfatide. That such a conversion does oceur was suggested by our early work with labeled galactose (37) and a recent report based on in vitro work (30). Presumably the conversion is by direct sulfation with phosphoadenosyl phosphosulfate.

\section{The Ganglioside Fatty Acids}

Virtually all the ganglioside FA are aecounted for as 16:0,18:0, and 20:0. These were examined as part of the 3-age turnover study described above (17). The curves for the specific activity of $16: 0$ showed a very rapid rise followed by a fairly rapid fall. The rate of fall dropped off with time, particularly with increasing age of the rat group. Comparison with the 16:0 of glyeerophosphatides showed quite similar drop-off rates and speeifle activities, so this sphingolipid is evidently much more active metabolically than most of the other glycolipids. A similar conclusion was drawn from our earlier study with galactose$\mathrm{C}^{14}(37)$. 
The 18:0 of ganglioside yielded curves similar to the 18:0 of glycerophosphatides. In both groups of acids (with only one exception) the curves were surprisingly like the theoretical precursor-product curves predicted by Zilversmit, Entenman, and Fishler (42). The 18:0 curves rose more slowly than the 16:0 curves, then the latter intersected the maximum of the 18:0 eurves, and then the two curves descended together (with the 18:0 now more radioactive). The curves are thus quite consistent with the elongation theory and evidence. The curves for 20:0 were similar to those for 18:0.

Although ganglioside deposition slows down somewhat after the rat reaches 21 days of age, the turnover curves show that ganglioside FA is being metabolized even at 52 days. (However, the 20:0 ganglioside appears to be inert after 32 days).

\section{General Conclusions from Turnover Studies}

The common belief that the brain FA are inert arose from two observations: (1) Labeled precursors injected systemically into animals gave rise to low activities in the brain lipids; (2) Long-term turnover studies of brain lipids have shown remarkably long persistence of radioactivity. The first type of observation was made without consideration of the bloodbrain barrier, which calls for the use of large doses of radioactivity in order to get measurable activities into the brain. The barrier, however, has little to do with the rate of brain metabolism; it simply reduces the choice of precursors which the brain can draw upon. The researchers who made these experiments did not appreciate the need to consider time as a variable in discussing reaction rates.

The second type of observation was made without examining the early time points so that the rapidly metabolizing fractions were missed. Unquestionably they point to the existence of a fraction in brain which metabolizes very slowly and perhaps not at all. This might well be myelin or some lipid structure concerned with the preservation of memory (37). Our shorter term experiments supplement the long term experiments and show that a portion of the brain lipid undergoes breakdown even during the period of rapid deposition. Indeed, the breakdown rates seem to be highest during the periods of most rapid synthesis or deposition. Lipid breakdown continues well after the period of most rapid myelination. At the moment there is no way to measure the relative amounts of the rapidly and slowly metabolizing fractions.
Our turnover and decarboxylation studies indicate the presence of several different metabolic pools and future work must examine the various subeellular regions separately.

\section{FINAL COMMENTS}

This paper on glyeolipid fatty acids was based primarily on the work of this laboratory and covers work by Drs. Amiya K. Hajra, W. Ewart Davies, Krystyna C. Kopaczyk, and S. S. Parmar. We are indebted to Dr. Bernard Agranoff, Coordinator of Biological Sciences in this Institute, for many valuable insights into our data. For reasons of space we have neglected to mention relevant work from other laboratories. Our critical comments are offered only to clarify certain approaches to the study of brain metabolism and will, we hope, be taken good-naturedly.

\section{ACKNOWLEDGMENT}

Supported in part by grant No. NB03192 from the Institute of Neurological Diseases and Blindness, USPHS.

\section{REFERENOES}

1. Brady, R. O. J. Biol Chem. 237, PC2416 (1962).

2. Brady, R. O., R. M. Bradley, and E. G. Trams. J. Biol. Chem. 235, $3093(1960)$

3. Brady, R. O., and G. J. Koval, J. Biol. Chem. $233,26(1958)$.

4. Bressler, R., and S. J. Wakil, Ibid. 236, 1643 (1961).

5. Carroll, K. K., J. Lipid Res. 2: 135, 1961.

6. Cleland, W. W., and E. P. Kennedy, J. Biol. Chem, $235,45(1960)$.

7. Davies, W. E., Y. Kishimoto and N. S. Radin, unpublished data.

8. Folch, J. M. Lees and G. H. Sloane Stanley, J. Biol. Chem. 226, 497 (1957).

9. Fulco, A. J., and J. F. Mead, Ibid. 236, 2416 (1961).

10. Hajra, A. K., and N. S. Radin, J. Lipid Res. 3, 327 (1962).

11. Hajra, A. K., and N. S. Radin, Ibid. 4, 270 (1963).

12. Hajra, A. K., and N. S. Radin, Ibid. 4, 448 (1963).

13. Harlan, W. R., Jr., and S. J. Wakil, J. Biol. Chem. 238, 3216 (1963)

14. Hitchcock, C., and A. T. James, Biochem. J.93 $22 \mathrm{P}$ (1964).

15. Holloway, P. W., and S. T. Wakil, J. Biol, Chem. $239,2489,(1964)$.

16. Jacobson, S., J. Comp. Neurol. 121, 5 (1963)

17. Kishimoto, Y., W. E. Davies and N. S. Radin

J. Lipid Res. 6, 525 (1965).

18. Kishimoto, $\mathrm{Y}$, and N. S. Radin, Tbid. 1, 72 (1959).

19. Kishimoto, Y., and N. S. Radin, Ibid. 1, 79

(1959).

20. Kishimoto, Y., and N. S. Radin, Ibid. 4, 130 (1963).

21. Kishimoto, $\mathrm{Y}$, and N. S. Radin, Ibid, 4, 437 (1963).

22. Kishinoto, Y., and N. S. Radin, Ibid. 4, 444 (1963).

23. Kishimoto, Y., and N. S. Radin, Ibid. 5, 94 (1964).

24. Kishimoto, Y., and N. S. Radin, Ibid, 5, 98 (1964)

25. Kishimoto, Y., and N. S. Radin, Ibid. 6, 435 (1965). 
zo. Kishimoto, Y., and N. S. Radin, Ibid. in press. 27. Levis, G. M., and J. F. Mead, J. Biol, Chem. 239, 77 (1964).

28. Lorch, E., S. Abraham and I. L. Chaikoff Biochim. Biophys. Acta 70,627 (1963).

29. Marsh, J. B., and A. T. James, Ibid. 60,320 (1962).

30. McKhann, G. M., R. Levy and W. Ho, Federation Proc. 24, 361 (1965).

31. Mead, J. F., and A. J. Fulco, Biochim. Biophys. Acta 54. 362 (1961)

32. Mead, J. F, and G. M. Levis, J. Biol, Chem. 238,1634 (1963).

33. O'Brien, J. S., Science 147, 1099 (1965)

34. Radin, N. S., JAOCS 42,569 (1965).

35. Radin, N. S., and Y. Akahori, J. Lipid Res. 2, 335 (1961).
36. Radin, N. S., F. B. Lavin and J. R. Brown, J Biol. Chem. 217, 789 (1955).

37. Radin, N. S., F. B. Martin and J. R. Brown, Ibid. 224, 499 (1957).

38. Reiner, J. M., Arch. Biochem. Biophys. 46, 53 (1953).

39. Rouser, G., G. Kritchevsky, D. Heller and E. Lieber, JAOCS 40, 425 (1963).

40. Rowe, O. E., Biochim. Biophys, Acta 84, 424 (1964).

41. Ställberg-Stenhagen, S., and L. Svennerholm, J. Lipid Res. 6, 146 (1965).

42. Zilversmit, D. B., O. Entenman and M. C. Fishler, J. Gen. Physiol. 26, 325 (1943).

[Received July 7, 1965] 